\title{
Main Trunk and Division Middle Cerebral Artery Occlusions: Differences in Recanalization Times, Number of Stent Retriever Passes and Clinical Outcomes: A Single-Center Experience
}

\author{
Ihtesham A. Qureshi Alberto Maud Salvador Cruz-Flores \\ Gustavo J. Rodriguez \\ Neurology Department, Texas Tech University Health Sciences Center, El Paso, Tex., USA
}

\author{
Key Words \\ Middle cerebral artery · Dominant division - Stent retriever - Main trunk - Recanalization · \\ Endovascular treatment
}

\begin{abstract}
Background and Purpose: In this article, we present our experience with the recanalization of the middle cerebral artery (MCA), we hypothesize that there are higher rates of recanalization with fewer stent retriever passes and better clinical outcomes in patients with division MCA occlusions. A more complex anatomy at the bifurcation may prevent a faster recanalization in main trunk MCA occlusions. Methods: We retrospectively identified consecutive patients admitted with MCA occlusions who underwent mechanical thrombectomy using stent retrievers. We categorized patients into division MCA and main trunk MCA occlusions based on angiography. Variables were compared between the groups. We further analyzed patients with trunk MCA occlusions to identify reasons for delays in recanalization. Results: There were 32 MCA occlusions that underwent mechanical thrombectomy and eligible for the analysis during the study period. Of those, 11 were main trunk MCA occlusions. Univariate analysis disclosed a trend toward a lower GP-to-recanalization time $(p=0.05)$ and a lower number of passes required for recanalization in division MCA occlusions. However, there was a significantly better outcome in patients with division MCA occlusion after multivariate analysis. Analyzing main trunk MCA occlusion data, we found that the need for more than one pass to achieve recanalization led to a trend toward a longer GP-to-recanalization time and a worse
\end{abstract}

All authors contributed equally to this study. 
outcome. When the stent was placed in the dominant division, the chances of recanalization were significantly higher. Conclusions: Division MCA occlusions have higher recanalization rates with fewer stent retriever passes and better clinical outcomes than main trunk MCA occlusions, likely due to a more favorable anatomy. Measures like placing the stent retriever in the dominant division may decrease recanalization times and improve clinical outcomes in main trunk MCA occlusions.

(C) 2015 S. Karger AG, Base

\section{Introduction}

Mechanical thrombectomy with stent retrievers, when performed in patients within the first few hours from symptom onset, is an effective therapy for ischemic stroke due to large vessel occlusion. The middle cerebral artery (MCA) is the most common vessel involved in ischemic stroke and the most commonly treated in endovascular trials [1-6]. Time to recanalization and quality of reperfusion are the strongest predictive variables of good clinical outcome after recanalization. As such, strategies directed to shorten the time from symptom onset to recanalization are needed [7].

We hypothesized that main trunk MCA occlusion has worse clinical outcomes, likely due to the higher number of passes needed and longer recanalization times. In addition, in main trunk MCA occlusion, the placement of the stent retriever in the dominant division of the MCA results in a faster recanalization. We report our experience with the endovascular treatment of MCA occlusions in a single center.

\section{Methods}

\section{Patient Population}

From a prospectively collected neurointerventional database at a single center that offers comprehensive stroke services (University Medical Center of El Paso, El Paso, Tex., USA), we retrospectively identified patients with occlusion of the MCA and selected those that underwent mechanical thrombectomy using stent retrievers. Data collection included information about age, gender, comorbidities (myocardial infarction, atrial fibrillation, dyslipidemia, diabetes mellitus, hypertension, history of alcohol abuse, cigarette smoking and previous history of stroke), body mass index (BMI), use of intravenous (IV) or intra-arterial (IA) recombinant tissue plasminogen activator (rt-PA), National Institute of Health Stroke Scale (NIHSS) score at presentation and 90-day modified Rankin Scale (mRS) score. The NIHSS and mRS scores were obtained by certified healthcare providers. Angiograms of patients with MCA occlusion were reviewed. Once the occlusion in the MCA was angiographically confirmed, patients were categorized into two different groups: patients with a main trunk MCA occlusion and those with an MCA division occlusion given the location of the thrombus. Variables were compared between the two groups.

In patients with main trunk MCA occlusion, further documentation of the stent retriever position (dominant vs. nondominant division) and whether recanalization was achieved with each stent retriever pass was collected. We then separated patients into two subgroups according to the number of passes required for recanalization, one pass versus more than one pass. Variables were compared between the two subgroups. We also compared cases in which recanalization was obtained with the stent retriever placed in the dominant versus nondominant division.

\section{Recanalization Therapies for Ischemic Stroke: Hospital Protocol}

If eligibility criteria were met, the IV rt-PA was administered at a standard dose of $0.9 \mathrm{mg} / \mathrm{kg}$ after noncontrast computerized tomography (CT) ruled out intracerebral hemorrhage. Endovascular treatment comprised mechanical thrombectomy usually within $6 \mathrm{~h}$ that could be supplemented with IA rt-PA. Patients were eligible for endovascular treatment on the clinical and neuroimaging basis and if they had an NIHSS score of $\geq 10$ or less and a vessel occlusion was documented on a CT angiogram. Wake-up stroke qualified for 
endovascular treatment if the ASPECTS (Alberta Stroke Program Early CT Score) was $\geq 7$. We did not exclude patients solely on the basis of age. However, we excluded patients with baseline dependency from endovascular treatment. Timelines within the angiography suite were recorded and included groin puncture (GP) time (when lidocaine was given), diagnostic angiography time (the time angiography shows the occlusion), microcatheter time (first microcatheter injection beyond the thrombus), and recanalization/final angiography time. A follow-up head CT was obtained in all patients $24-36 \mathrm{~h}$ after recanalization treatments. Symptomatic hemorrhage was defined as a hemorrhage causing clinical worsening of the NIHSS $\geq 4$ points. Carotid terminus occlusion that could have extended to the MCA or tandem occlusions affecting the MCA were excluded, since additional treatments and longer times were expected.

\section{Endovascular Technique}

In our institution, it is the routine practice to attempt endovascular treatment under moderate sedation, if not possible, the case is then converted into general anesthesia. An 8-Fr balloon guide catheter is placed in the postbulbar internal carotid artery. A 5-Fr 125-cm Vert catheter (Impress Diagnostic, Jordan, Utah, USA) or a $130-\mathrm{cm}$ Simmons 2 catheter (Soft-Vu, Latham, N.Y., USA) is used to select the cervical internal carotid artery and advance the 8-Fr balloon catheter. A diagnostic angiogram is performed through the 8-Fr balloon guide catheter. The most common microcatheter used is a Prowler select plus (Codman Neurovascular, Miami, Fla., USA) over a Synchro 2 (Boston Scientific, Natick, Mass., USA) microwire. Once the thrombus is crossed, a microcatheter angiogram is performed, confirming its proper placement. Stent retrievers approved in the US are used at our institution and a maximum of three passes are allowed for each vessel. Adjuvant IA rt-PA may be considered by the treating operator throughout the procedure, and in case of mechanical thrombectomy failure, it is up to the operator to abort the case or use other mechanical devices such as the aspiration system. The final Thrombolysis in Cerebral Infarction (TICI) score is recorded.

\section{MCA Anatomy}

The MCA is perhaps the most complex artery of the brain and there is no strong consensus in its nomenclature. We considered an occlusion of the main trunk MCA if the occlusion was at its main branching point. Usually the main trunk or stem of the MCA bifurcates in two divisions or subsequent trunks, known as superior and inferior. There is also an intermediate trunk that most of the times arises from the inferior division. Occasionally, the intermediate trunk arises early enough and resembles a trifurcation (pseudotrifurcation). The division from which the intermediate trunk arises is considered the dominant division and counts with a larger diameter. The precentral, central and postcentral (also known as the anterior parietal) arteries usually arise from the intermediate trunk. These branches give the blood supply to the primary somatosensory cortex [8].

\section{Statistical Analysis}

This is a convenience sample considering that we included those patients available in our dataset. All variables were compared using cross-tabulations between occlusion location (main trunk MCA vs. division MCA) and further a subgroup in the main trunk MCA occlusion (single pass of the stent retriever vs. more than one pass). In addition, comparison was made regarding the recanalization obtained with placement of the stent retriever in the dominant or nondominant division. All data was descriptively presented using mean \pm standard deviation (SD) for continuous and frequencies for categorical variables. The differences between the two groups were assessed using univariate analysis, Student's $t$ test for continuous variables and $\chi^{2}$ test for categorical variables. If there was a difference, multivariate analysis was performed to adjust for those variables. A p $<0.05$ value was considered to be statistically significant. All statistical analysis was performed using IBM SPSS version 19.0 software.

\section{Results}

A total of 72 patients underwent endovascular treatment during the study period (April 2013 to July 2015). There were 35 MCA occlusions identified. Other cases were tandem occlusions $(n=13)$, carotid terminus occlusions $(n=8)$, basilar artery occlusions $(n=5)$, cervical carotid occlusions $(n=4)$, intracranial atherosclerosis $(n=4)$ and other $(n=3)$. Out of the 35 patients with MCA occlusion, mechanical thrombectomy was not technically possible in 3 
Table 1. Demographics and clinical characteristics: comparison between main trunk and division MCA occlusions

\begin{tabular}{l|l}
\hline DOI: $10.1159 / 000442578$ & $\begin{array}{l}\text { @ } 2015 \text { S. Karger AG, Basel } \\
\text { www.karger.com/ine }\end{array}$ \\
\hline
\end{tabular}

Qureshi et al.: Thrombectomy in Middle Cerebral Artery Occlusion

\begin{tabular}{lccl}
\hline Variables & Main trunk & Division & p \\
& MCA & MCA \\
occlusion & occlusion & \\
& 11 & 21 & \\
\hline Total cases & $6(18)$ & $12(35)$ & n.s. \\
Females & $71.00 \pm 14.4$ & $70.24 \pm 15$ & n.s. \\
Age, years & $1(2.9)$ & $2(5.8)$ & n.s. \\
Myocardial infarction & $6(17.6)$ & $11(32.4)$ & n.s. \\
Atrial fibrillation & $8(23.5)$ & $6(17.6)$ & n.s. \\
Dyslipidemia & $7(20.6)$ & $7(20.6)$ & n.s. \\
Diabetes mellitus & $11(32.4)$ & $17(50)$ & n.s. \\
Hypertension & $1(2.9)$ & $3(8.7)$ & n.s. \\
H/O smoking & $1(2.9)$ & $4(11.6)$ & n.s. \\
H/O alcohol & $7(20.5)$ & $9(26.4)$ & n.s. \\
H/O previous stroke & $29.25 \pm 4.16$ & $29.09 \pm 5.57$ & n.s. \\
BMI & $4(11.4)$ & $2(5.7)$ & n.s. \\
IV rt-PA use & $3(8.5)$ & $11(31.4)$ & n.s. \\
IA rt-PA use & $21 \pm 5.55$ & $13.86 \pm 5.74$ & $0.002^{*}$ \\
Initial NIHSS score & $1.8 \pm 0.8$ & $1.4 \pm 0.7$ & 0.1 \\
SR passes & $10(91)$ & $21(100)$ & n.s. \\
Final TICI score of $2 \mathrm{~b} / 3$ & $60.64 \pm 25.48$ & $44.29 \pm 19.90$ & 0.05 \\
GP-to-recanalization (min) & $3.18 \pm 2.24$ & $1.53 \pm 1.28$ & $0.02^{*}$ \\
mRS score & & & \\
\hline
\end{tabular}

Values are expressed as n (\%) or mean \pm SD. H/O = History of; SR = stent retriever; n.s. $=$ not significant. $*$ Statistically significant.

patients due to difficulties with carotid catheter access secondary to arch tortuosity. In the final analysis, 32 patients were studied, 11 with main trunk MCA occlusion and 21 with division MCA occlusion. Three patients required general anesthesia with mechanical ventilation, one of them in the main trunk MCA occlusion group. Six patients had wake-up strokes, three in each group. No symptomatic hemorrhage was observed in either group. When these two groups were compared, we found differences in the initial NIHSS score, which was significantly higher in the main trunk MCA occlusion. There was a trend toward a greater number of stent retriever passes and a longer GP-to-recanalization time in patients with trunk MCA occlusion. There was a statistically significant worse outcome at 3 months in the main trunk MCA occlusion group (table 1) that remained after multivariate analysis was performed adjusting for the initial NIHSS ( $\mathrm{p}<0.01)$.

When we analyzed and compared the subgroups of patients with main trunk MCA occlusion requiring one pass of the stent retriever versus more than one pass for recanalization, we found a trend toward a higher GP-to-recanalization time in patients requiring more than one pass ( 39.8 vs. $69.1 \mathrm{~min}, \mathrm{p}=0.05$, respectively), with a trend toward a worse outcome at 3 months. Out of all patients with main trunk MCA occlusion, 10 had a final TICI score of $2 \mathrm{~b} / 3$ and 1 had a final TICI score of $2 \mathrm{a}$. Five of the patients were recanalized with the first pass.

There were 21 passes in the 11 patients with main trunk MCA occlusion. With the first pass, in all recanalized cases the stent retriever was placed in the dominant division; however, there were 2 additional patients that did not recanalize despite having the stent retriever placed in the dominant division. With the second pass, 2 patients in whom the stent retriever was placed in the dominant division did not recanalize and in 4 patients the stent retriever was placed in the nondominant division but only 1 recanalized. With the third pass, 4 patients had the stent retriever placed in the dominant division and only 1 did not recanalize. This last 
Table 2. Number of passes accomplished with stent retrievers in patients with main trunk MCA occlusion

\begin{tabular}{l|l}
\hline DOI: $10.1159 / 000442578$ & $\begin{array}{l}\text { C } 2015 \text { S. Karger AG, Basel } \\
\text { www.karger.com/ine }\end{array}$ \\
\hline
\end{tabular}

Qureshi et al.: Thrombectomy in Middle Cerebral Artery Occlusion

\begin{tabular}{lll}
\hline & Recanalization, $\mathrm{n}$ & No recanalization, $\mathrm{n}$ \\
\hline Dominant & 8 & 5 \\
Nondominant & 1 & 7
\end{tabular}

Comparison of the recanalization obtained according to the placement of the stent retriever in the dominant versus nondominant division of the MCA in up to three passes per patient. The $\mathrm{p}$ vaule was 0.02 and was statistically significant.

patient had rescue recanalization with the aspiration system. We observed that the chances of obtaining recanalization were significantly higher when the stent retriever was placed in the dominant division in up to three passes (table 2).

\section{Discussion}

Patients with main trunk MCA occlusion needed more stent retriever passes to achieve recanalization, with longer GP-to-recanalization times and worse clinical outcomes compared to division MCA occlusion. In addition, in patients with main trunk MCA occlusion, recanalization was more often achieved when the stent retriever was placed in the dominant division using up to three passes. Our findings suggest that efforts should be made to systematically favor the microcatheterization of the dominant division in main trunk MCA occlusion. It may increase the rate of successful recanalization with fewer number of stent retriever passes and shorter GP-to-recanalization times. This approach may also have an impact on the clinical outcome of these stroke patients.

During endovascular treatment of a main trunk MCA occlusion, it is difficult to visualize the dominant division, as we have poor or no contrast opacification. The trajectory of the microwire under fluoroscopic guidance in the lateral view may be helpful to determine if the intermediate trunk (pointing to the central region) is being selected. The subsequent microcatheter angiogram will help with the confirmation that the dominant division of the MCA has been catheterized. If the intermediate trunk cannot be selected, the operator should aim for the selection of a branch of the inferior division. It has been reported that the inferior division is most commonly the dominant division counting with the largest diameter [8].

We observed that recanalization of the main trunk MCA occlusion was obtained with fewer passes when the stent retriever was placed in the dominant division. The reason for these results may be due to the dominant division having a larger diameter and burden of the thrombus. When the stent retriever is placed in the dominant division, a larger surface of the stent is in contact with a larger surface of the thrombus providing a better capture profile (fig. 1).

In our study, we used stent retrievers approved and available in the United States. Those are the Trevo (Trevo XP ProVue Retriever-Stryker) and the Solitaire (Solitaire FR DeviceEV3). In a swine and canine animal model, the Trevo stent retriever achieved $100 \%$ recanalization with a TICI score of 3 , and a total of 15 cases required just one stent retriever pass and 1 case required two passes. Similarly, in another animal model, the Solitaire stent retriever achieved $100 \%$ recanalization with a TICI score of 3, recanalization with one pass was obtained in 5 cases, and two passes were required in 1 case $[9,10]$. The anatomy of the animal models appears to resemble that of the division MCA in humans without major branching points, which supports our results. We achieved $100 \%$ recanalization of the division MCA occlusions with an average slightly above one stent retriever pass per case. 

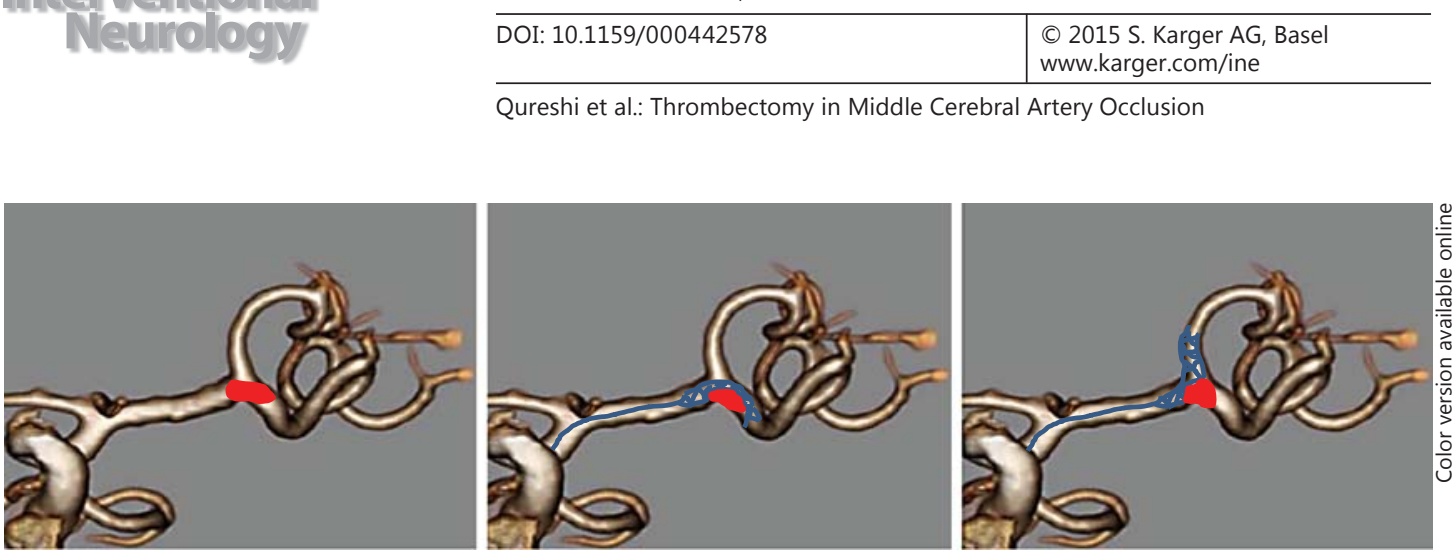

Fig. 1. Occlusion of the main MCA trunk is shown. a The thrombus is located in the distal MCA, at the bifurcation point. $\mathbf{b}$ The stent retriever is placed in the dominant division, in this case, the inferior division. A larger surface of the stent retriever is in contact with the thrombus and its complete retrieval is more likely to occur. c The stent retriever is placed in the nondominant division, in this case, the superior division. There is less contact with the thrombus, the thrombus may be further pushed into the dominant division and its complete retrieval is less likely to occur.

Over the last few years, a substantial effort has been directed toward improving the technology and technique of endovascular treatment for acute ischemic stroke. However, timelines in the endovascular treatment of ischemic stroke remained unaddressed. As of today, the only time goal with recanalization therapies for the treatment of acute ischemic stroke is the door-to-needle time. Improvement of this time has shown to result in better clinical outcomes [11]. Our observation may help shorten the times to recanalization and potentially improve the clinical outcome.

The main limitations of our study are the retrospective design and a single-center experience, which decreases the power of the study to detect differences in GP-to-recanalization time and overall outcome of the patient. Therefore, a pooled data study with a larger sample size is warranted to confirm and strengthen our results.

\section{Conclusions}

Our study shows that division MCA occlusions have higher recanalization rates with fewer stent retriever passes needed to achieve recanalization and better clinical outcomes than main trunk MCA occlusions. It further suggests that in main trunk MCA occlusions, the placement of the stent retriever in the dominant division may decrease the number of passes required to achieve recanalization, the GP-to-recanalization and may improve the clinical outcome. Larger studies are required to confirm our findings.

\section{Disclosure Statement}

The authors report no conflicts of interest.

\section{References}

1 Goyal M, Demchuk AM, Menon BK, et al: Randomized assessment of rapid endovascular treatment of ischemic stroke. N Engl J Med 2015;372:1019-1030.

2 Campbell BC, Mitchell PJ, Kleinig TJ, et al: Endovascular therapy for ischemic stroke with perfusion-imaging selection. N Engl J Med 2015;372:1009-1018. 
3 Berkhemer OA, Fransen PS, Beumer D, et al: A randomized trial of intraarterial treatment for acute ischemic stroke. N Engl J Med 2015;372:11-20.

4 Jovin TG, Chamorro A, Cobo E, et al: Thrombectomy within $8 \mathrm{~h}$ after symptom onset in ischemic stroke. $\mathrm{N}$ Eng J Med 2015;372:2296-2306.

5 Saver JL, Goyal M, Bonafe A, et al: Stent-retriever thrombectomy after intravenous t-PA vs. t-PA alone in stroke. N Eng J Med 2015;372:2285-2295.

6 Rordorf G, Koroshetz WJ, Copen WA, et al: Regional ischemia and ischemic injury in patients with acute middle cerebral artery stroke as defined by early diffusion-weighted and perfusion-weighted MRI. Stroke 1998;29: 939-943.

7 Khatri P, Abruzzo T, Yeatts SD, et al: Good clinical outcome after ischemic stroke with successful revascularization is time-dependent. Neurology 2009;73:1066-1072.

8 Kahilogullari G, Ugur HC, Comert A, et al: The branching pattern of the middle cerebral artery: is the intermediate trunk real or not? An anatomical study correlating with simple angiography. J Neurosurg 2012;116: 1024-1034.

9 Jahan R: Solitaire flow restoration device for treatment of acute ischemic stroke: safety and recanalization efficacy study in a swine vessel occlusion model. AJNR Am J Neuroradiol 2010;31:1938-1943.

10 Nogueira RG, Levy EI, Gounis M, et al: The Trevo device: preclinical data of a novel stroke thrombectomy device in two different animal models of arterial thrombo-occlusive disease. J Neurointervent Surg 2012;4: 295-300.

11 Fonarow GC, Smith EE, Saver JL, et al. Timeliness of tissue-type plasminogen activator therapy in acute ischemic stroke: patient characteristics, hospital factors, and outcomes associated with door-to-needle times within 60 min. Circulation 2011;123:750-758. 